\title{
The preparedness of the radiology department at Damascus Hospital during COVID- 19 lockdown
}

\begin{abstract}
The outbreak of the Coronavirus COVID-19 pandemic originated in China Wuhan province in December 2019, China and it causes severe acute respiratory and lung infection. Up to this date, over 5 million people around the world especially in Italy, USA and Iran have been infected with the virus.

The Syrian government represented in Ministry of Health and other health authorities have responded to this outbreak with lockdown of the public and private activities among many other procedures. These responses are led by public health authorities in coordination with the hospitals in the Syrian cities including Damascus Hospital which belongs to Ministry of Health. Damascus hospital and other hospitals which belong to Ministry of Health continued to receive referrals of patients during the lockdown period for their diagnosis. As Damascus hospital is considered the largest hospital, the review will focus on services that had been offered by it.
\end{abstract}

Keywords: COVID-19, pandemic, severe acute respiratory infection, ministry of health, SARS-CoV-2
Volume 7 Issue 6 - 2020

\author{
Abear Labban,' 'Wassim Bin Khadra \\ 'Radiology resident, Damascus Hospital, Syria \\ ${ }^{2} \mathrm{Head}$ radiology Department, Damascus Hospital, Syria
}

Correspondence: Abear Labban, Radiology resident, Damascus Hospital, Damascus. Syria, Email drluay@gmail.com

Received: November 12, 2020 | Published: December 22, 2020

\section{Introduction}

Since its emergence in November 2019 in Wuhan, China, the COVID-19 pandemic caused by the SARS-CoV-2 virus has spread rapidly across the world in a matter of months, affecting 212 countries and territories, with more than 3.8 million cases and 265,000 deaths worldwide as of 7 May 2020. ${ }^{1}$ In the United Kingdom there have been over 200,000 cases and 30,000 deaths as of 7 May 2020. Chest radiograph is a primary diagnostic tool alongside clinical assessment and lab parameters, while $\mathrm{CT}$ has been primarily reserved for complications and problem solving. ${ }^{2}$ The infectious potential of SARS-CoV-2 means that leaders must prepare appropriately in order to safely practice radiology. ${ }^{3}$ We describe our experience of preparedness for the COVID-19 pandemic at Damascus Hospital.

Damascus Hospital (aka Al Mujtahid Hospital) is located in Damascus city and is considered one of the largest hospitals in Syria which contains 645 beds, 36 of which are used for intensive care. It was founded in 1947 and is run by the Ministry of Health. This hospital has a special emergency ward for internal diseases. The hospital provides medical, therapeutic and surgical services to patients. The hospital is also used to train students and graduate doctors from Syrian universities and contributes to medical scientific research. The activities of the hospital include internal specialties, including cardiac, neurological, and gastrointestinal and kidney. It also performs general, endoscopic, vascular and cardiac surgery, neurosurgery and urinary surgery. It has a kidney transplant unit and a physiotherapy unit. The hospital also has a modern laboratory for specimen's analysis which includes hormonal, immune, tumor, pharmaceutical, and lymphatic tests.

Among the other departments, the hospital has radiology department which has facilities for MRI and CT scan, a gamma camera and lithotripsy device. In this department, there are 3 consultants and 21 residents who are doing 4 years internship to become consultants and they are being rotated in the facilities of the department. In addition, there are 11 radiology technicians who perform the daily work at the department. The hospital administration has put a set of policies and procedures for this department as radiology preparedness instructions during emergencies such as COVID-19 outbreak in order to achieve sufficient capacity for continued operation and to maintain radiologic diagnostic and interventional support for the entirety of the hospital and health system.

We redesigned our service to support the hospital with access to an efficient imaging service and senior-led opinion. To achieve this, our department has made several changes in reporting, vetting and work practices. ${ }^{4}$ The main activity of the department of diagnostic radiology at Damascus Hospital is the interpretation and analysis of the medical imaging which is then reported to each medical department. Advances in diagnostic radiology are remarkable and the clinical significance of such diagnosis increases every day. Thus, our department provides key support to the diagnostic activities.

The characteristics of a radiology department are determined by the roles and functions of the hospital and the needs of the community it serves. ${ }^{5}$ If these facilities are part of a large teaching institution, some departments may have teaching and research in addition to patient care responsibilities. In smaller hospitals, the radiology department may be involved only in patient care.

The organization of a radiology department affects its internal structure and the disposition and management of personnel and fiscal resources. ${ }^{6}$ Management goals are to arrange employees into working groups according to their work functions. Administration directs the efforts and skills of employees toward reaching departmental objectives in a cohesive and satisfying fashion. Specialized areas within the radiology department include diagnostic radiology.? Currently, the sections of radiology departments that are devoted to diagnostic services only are often called imaging departments. This 
article is devoted almost exclusively to the diagnostic radiology department because it is very important and plays a role in diseases diagnostic process.

The head of the radiology department has the following duties:

1. Contribution in medical activities as required

2. Establishment of an effective working relationship with the medical staff, the administration, and other departments and services

3. Development and approval of all policies and procedures for the radiology department

4. Verification of the qualifications and capabilities of all radiology staff technical personnel

5. Development of comprehensive safety rules in cooperation with the hospital safety committee

6. Review and evaluation of the quality and appropriateness of radiologic services

7. Advisement of the medical staff and administration of equipment needs, modification, and utilization

The definitive objective of the diagnostic radiology department is to support physicians in their efforts to diagnose and treat disease by providing them with timely and reliable information obtained from radiographic examinations using the least amount of radiation necessary for conducting the radiographic examination. ${ }^{8}$

To ensure the reliability of this diagnostic information, careful attention must be given to the performance of every examination, beginning when the examination is ordered and continuing until the examination results have been returned to the requesting physician. This relentless attention must come from all members of the radiology staff. Diagnostic radiology services should be conveniently available to meet the needs of the patient and should be directed by one or more qualified radiologists and a sufficient number of qualified technical personnel.

Because patient care is the primary concern of any hospital department and effective patient care depends on cooperation between all of the hospital departments, the radiology department must have an effective working relationship with the medical staff, the administration, and other hospital departments and services.

The department is also important for members of the radiology department so that the process of patient care runs as smoothly as possible. The radiology staff must interact with the personnel department, which is largely responsible for recruiting personnel and maintaining personnel records. Radiology services are often a substantial part of the patient's hospital expense; therefore department personnel need to be familiar with business services, which is the department that monitors billing procedures.

Developing and approving all radiology department policies and procedures is the responsibility of the radiology administrator. When this responsibility is executed thoroughly, the radiology department should function in a smooth and organized manner.

The aim of this study was to examine the number of patients referred to the radiology department and the facilities used during the period of March 2020 and May 2020 which represent the total governmental lockdown period.

\section{Materials and methods}

During this study, we reviewed the admission lodge of the radiology department and collected information about the people who registered in the department during the lockdown period due to Covid-19 pandemic which was between March and May 2020. The total number of admission was 503 distributed among males and females and among several age groups.

\section{Results}

The results of this review have shown that there were 233 males $(46.3 \%)$ and 270 females $(53.7 \%)$. Their ages and their percentages are demonstrated in Table 1.

Table I The number of males and females registered at the radiology department

\begin{tabular}{lll}
\hline Gender & Number & $\%$ \\
\hline Males & 233 & 46.3 \\
Females & 270 & 53.7 \\
Total & 503 & 100
\end{tabular}

The patients who referred to the radiology department varied in their ages. They were divided into several age groups according to their age. There were 62 children patients $(12.3 \%)$ in the first age group (Birth to 5 years). In the second age group (6-15 years) there were 75 patients $(14.9 \%)$ whereas in the age group 16-30 years, there were 85 patients $(16.9 \%)$. The highest number of patients was in the age group 31-50 years which has 166 patients (33\%) followed by the group of ages over 51 which have 155 patients $(22.9 \%)$. The numbers of patients and their percentages are shown in Table 2.

Table 2 Age groups of patients and their percentages

\begin{tabular}{lll}
\hline Age & Number & $\%$ \\
\hline Birth to 5 years & 62 & 12.3 \\
6-15 years & 75 & 14.9 \\
I6-30 years & 85 & 16.9 \\
3 I-50 & 166 & 33 \\
More than 5 I & 115 & 22.9 \\
Total & 503 & 100 \\
\hline
\end{tabular}

During the period of the lockdown, the radiology department performed different imaging types. The number of lumber imaging cases was the highest. They comprised $38.8 \%$ of the total imaging cases or 195 patients. Followed by brain imaging 159 patients (31.6 $\%)$ followed by mixed imaging 58 patients $(11.5 \%)$, cervical imaging 55 patients $(10.9 \%)$ and knee imaging 11 patients or $2.2 \%$. Finally, the numbers of patients were equal in pelvis, thoracic and pituitary imaging for 5 patients or $1 \%$ of the total patients. The results are demonstrated in Table 3. 
Table 3 Number and percentages of different types of imaging

\begin{tabular}{lll}
\hline Region & Number & $\%$ \\
\hline Lumbar & 195 & 38.8 \\
Pelvis & 5 & $\mathrm{I}$ \\
Thoracic & 5 & $\mathrm{I}$ \\
Extremities & 10 & 2 \\
Knee & $\mathrm{II}$ & 2.2 \\
Pituitary & 5 & $\mathrm{I}$ \\
Brain & 159 & 31.6 \\
Cervical & 55 & 10.9 \\
Mixed imaging & 58 & 11.5 \\
Total & 503 & 100 \\
\hline
\end{tabular}

Some types of imaging required contrast to reach a diagnosis. Lumbar imaging without contrast comprised $46.5 \%$ or 195 patient whereas 5 patients or $1.2 \%$ of lumbar imaging cases required contrast procedure. With regard to brain imaging 130 patients or $31.8 \%$ did not require contrast comparing to 29 patients or $7.1 \%$ had contrast. Cervical imaging with contrast had 5 patients or $1.2 \%$ compared with 50 patients or $12.2 \%$ did not require contrast as shown in table 4 .

Table 4 Number and percentages of imaging cases with and without contrast

\begin{tabular}{llll}
\hline Region & Contrast & Number & $\%$ \\
\hline \multirow{2}{*}{ Lumbar } & With contrast & 5 & 1.2 \\
& Without contrast & 190 & 46.5 \\
\multirow{2}{*}{ Brain } & With contrast & 29 & 7.1 \\
& Without contrast & 130 & 31.8 \\
Cervical & With contrast & 5 & 1.2 \\
Total & Without contrast & 50 & 12.2 \\
\hline
\end{tabular}

\section{Conclusion}

Our investigative study found that the operational management and accountability arrangements for radiology services were clear and appropriate. The study found that nearly all health boards are taking positive steps to reduce inappropriate referrals, but sign posting to local referral guidance could be improved. However, since our review, access to national referral guidance has improved.

The main problems which can be experienced in the activities of the radiology department can be summarized as the lack of integration between the departments; improving through implementation of subspecialized divisions and integrated calls. Lack of support for education and research and lack of replacement spare parts for Computerized Tomography Scan machine.

\section{Acknowledgments}

The authors would like to thank the staff of the admission department for keeping the records of patients admitted to the radiology department during the lockdown period due to Covid-19 pandemic.

\section{Conflicts of interest}

None.

\section{References}

1. Worldometer. COVID-19 coronavirus pandemic. 2020

2. London datastore. Coronavirus (COVID-19) deaths. 2020.

3. British Society of Thoracic Imaging. Thoracic imaging in COVID-19 Infection: Guidance for the reporting radiologist. BSTI. 2020.

4. British Society of Thoracic Imaging. Radiology decision tool for suspected COVID-19. 2020.

5. British Society of Thoracic Imaging and British Society of Gastrointestinal and Abdominal Radiology. Covid-19: BSTI/BSGAR decision tool for chest imaging in patients undergoing $\mathrm{CT}$ for acute surgical abdomen.

6. British Society of Gastrointestinal and Abdominal Radiology. CT Colonography activity and Covid-19: British Society of Gastrointestinal and Abdominal Radiology guidance. 2020.

7. NHSX. COVID-19 information governance advice for staff working in health and care organisations.

8. NHS England. Clinical guide for the management of radiology patients during the coronavirus pandemic (version 1). 